\title{
Influência da presença da fêmea sobre as características seminais do curimbatá (Prochilodus marggravii Walbaum, 1972)
}

\section{Influence of female presence on seminal characteristics of curimbatá (Prochilodus marggravii)}

\author{
Eduardo Shimoda ${ }^{1}$, Dálcio Ricardo de Andrade ${ }^{2}$, Manuel Vazquez Vidal Junior ${ }^{2},{ }^{3}$, José Frederico Stradiotti Silva ${ }^{2}$, \\ Oscar W.F. Carballo², Gilson Mendes da Cruz ${ }^{2}$
}

\section{Resumo}

Este trabalho foi realizado com o objetivo de se verificar a influência da fêmea sobre a produção seminal nos curimbatás. Foram selecionados nove machos (com alta produção seminal) e uma fêmea (com elevado grau de maturação gonadal) que foram acondicionados nos tanques $\mathrm{A}$ (cinco machos com peso médio de $142 \pm 27,1 \mathrm{~g} \mathrm{e}$ $19,4 \pm 1,64 \mathrm{~cm}$ de comprimento) e $\mathrm{B}$ (quatro machos de $22,8 \pm 3,42 \mathrm{~cm}$ de comprimento e $245 \pm 97 \mathrm{~g}$ de peso médio e uma fêmea). Decorridas 250 horas.graus, procedeu-se à coleta e à análise do sêmen, seguindo-se a hipofisação. Após outro período de 250 horas.grau, o sêmen foi novamente coletado e analisado. Foi verificada significativa e positiva influência da fêmea (ao nível de $5 \%$ de probabilidade) sobre a concentração espermática e sobre o número de espermatozóides vivos por $\mathrm{kg}$ de peso vivo. Turbilhonamento, percentual de espermatozóides vivos e volume seminal por peso de peixe não foram estatisticamente influenciados pela presença da fêmea.

Palavras chave: sêmen; peixe; Prochilodus marggravii; influência da fêmea

\section{Introdução}

Peixes reofílicos são caracterizados por realizarem migração reprodutiva. Este fenômeno, também conhecido como piracema, participa no processo de desenvolvimento gonadal (aumento na produção espermática e maturação final, ovulação e desova). Em tanques de piscicultura, esta migração fica impossibiltada e a reprodução apenas é viável mediante a utilização de técnicas de propagação artificial, como por exemplo a hipofisação.

A técnica da hipofisação consiste na aplicação de hormônios oriundos da hipófise de peixes doadores ou produzidos industrialmente. O seu procedimento sugere a utilização do sêmen de dois a três machos por desova de fêmea, o que garantiria um bom percentual de fertili- zação dos ovócitos (Woynarovich e Horvath, 1983; Valencia e Puentes, 1989). Entretanto, a literatura não especifica se reprodutores machos e fêmeas devem permanecer juntos ou em tanques separados durante todo $o$ processo de reprodução induzida.

Apesar de existirem vários trabalhos em outros países enfatizando o papel de feromônios na reprodução de peixes (Billard et al., 1989; Weerd e Richter, 1991; Defraipont e Sorensen, 1993; Stacey et al., 1994; Sorensen et al., 1995), no Brasil, os trabalhos relativos ao estudo do sêmen de peixes (Coser at al., 1984, 1987; Silveira et al., 1985, 1987, 1988; Kavamoto et al., 1989; Carolsfield et al., 1990) nada citam a respeito da influência da presença da fêmea sobre a produção de sêmen dos reprodutores durante o processo de desova induzida.

O objetivo deste trabalho foi verificar a influência da fêmea sobre a produção espermática do Prochilodus marggravii.

\section{Material e Métodos}

Este trabalho foi conduzido na Estação de Hidrobiologia e Piscicultura da Universidade Federal de Viçosa, Viçosa, MG, em dezembro de 1994, durante a estação reprodutiva. Foram selecionados nove machos (com alta produção seminal) e uma fêmea (com elevado grau de maturação gonadal), que foram distribuídos em dois tanques de alvenaria, no laboratório:

Tanque A: cinco machos (peso médio de $142 \pm 27,1$ g e 19,4 $\pm 1,64 \mathrm{~cm}$ de comprimento)

Tanque B: quatro machos (peso médio de $245 \pm 97 \mathrm{~g}$ e 22,8 $\pm 3,42 \mathrm{~cm}$ de comprimento) e uma fêmea.

Os exemplares permaneceram nos tanques $A$ (ausência de fêmea) e B (em presença de fêmea) por 250 horas.grau, procedendo-se, então, à coleta e à análise de sêmen. A seguir, os exemplares machos dos dois tanques foram hipofisados $(0,5 \mathrm{mg} / \mathrm{kg}$ de peso vivo) segundo Woynarovich e Horvath (1983). Os peixes permaneceram nos tanques por mais 250 horas.grau e foram no-

\footnotetext{
1 Mestrando em Produção Animal da UENF

2 Universidade Estadual Norte Fluminense, UENF, Av. Alberto Lamego, 2000, 28015-620 Campos dos Goytacazes, RJ, Brasil

${ }^{3}$ Doutorando em Zootecnia, Universidade Federal de Viçosa
} 
vamente extrusados e o sêmen analisado.

O volume do sêmen foi determinado através da observação direta na seringa graduada onde foi coletado. Para avaliação da percentagem de espermatozóides vivos foi utilizada a técnica da coloração diferencial com eosinanigrosina, descrita por Blom (1950), seguida da confecção de um esfregaço para observação e contagem em microscopia convencional (Fribourgh, 1966). O valor do turbilhonamento foi atribuído, subjetivamente, numa escala de 1 a 5 , em função da quantidade e vigorosidade das ondas de evolução espermática (Fonseca et al., 1992) observadas em aumento de 100 vezes (microscopia convencional), após ativação dos espermatozóides com água. Para determinação da concentração espermática, procedeu-se à diluição do sêmen (1:1000) em solução de formolsalina (Hancock, 1957), sendo a contagem realizada posteriormente em câmara hematimétrica de Neubauer "improved" (Silveira et al., 1987).

Foram obtidas as médias dos dados coletados que foram comparadas entre os tratamentos $(P<0,05$, teste F), segundo Pimentel Gomes (1966).

\section{Resultados}

Os dados relativos à análise seminal estão relacionadas nas Tabelas 1 (médias, coeficientes de variação, valores máximos e mínimos) e 2 (médias e significâncias).

\section{Discussão e Conclusão}

Pode-se observar nas Tabelas 1 e 2 que a concentração de espermatozóides/ml de sêmen foi significativamente mais elevada nos peixes colocados em presença da fêmea, independentemente deles estarem hipofisados ou não. Esta mesma influência da fêmea se fez nítida, e de forma positiva, sobre o número total de espermatozóides vivos por peso de peixe. O turbilhonamento e o percentual de espermatozóides vivos não foram estatisticamente diferentes nos tratamentos. Com relação ao volume de sêmen por peso, apesar dos valores médios serem mais elevados nos peixes em contato com a fêmea, a análise estatística também não mostrou diferença significativa entre os tratamentos.

Os feromônios sexuais, segundo a literatura, têm um efeito positivo sobre a reprodução dos peixes. Neste experimento, percebe-se a influência da presença da fêmea refletindo sobre o número de espermatozóides vivos por peso de peixe (Tabela 2). Segundo Billard et al. (1989), ocorre um aumento do volume de sêmen em carpas (Cyprinus carpio) em presença de fêmeas ovulando. $\mathrm{O}$ feromênio esteróide 17,20 B-P, produzido pelas fêmeas do goldfish (Carassius auratus), promoveu aumentos na produção espermática nos machos da mesma espécie (Sorensen et al., 1995) e nos machos de C. carpio (Stacey et al., 1994). Defraipont e Sorensen (1993) afirmam ainda que o feromônio 17,20 B-P aumenta a duração da motilidade espermática, embora nada seja conhecido a respeito do mecanismo promotor deste efeito.

Com base nos resultados obtidos neste experimento, podemos concluir que a presença da fêmea junto com os machos de $P$. marggravii afetou positivamente a concentração e o número de espermatozóides vivos por peso de peixe, contribuindo assim para um melhor perfil espermático de curimbatás submetidos ao processo de desova induzida em laboratório.

Tabela 1 - Médias relativas às análises seminais nos tratamentos $A$ e $B$

\begin{tabular}{|c|c|c|c|c|c|c|c|c|c|}
\hline \multirow[t]{3}{*}{$\therefore$} & & \multicolumn{4}{|c|}{ Ausência da fêmea } & \multicolumn{4}{|c|}{ Presença da fêmea } \\
\hline & & \multirow[t]{2}{*}{$x$} & \multirow{2}{*}{$\begin{array}{l}\text { CV } \\
(\%)\end{array}$} & \multicolumn{2}{|c|}{ Valores } & \multirow[t]{2}{*}{$x$} & \multirow{2}{*}{$\begin{array}{l}\text { CV } \\
(\%)\end{array}$} & \multicolumn{2}{|c|}{ Valores } \\
\hline & & & & Max. & Min. & & & Max. & Min. \\
\hline \multirow{2}{*}{\multicolumn{2}{|c|}{$\begin{array}{l}\text { Comprimento }(\mathrm{cm}) \\
\text { Peso }(\mathrm{g})\end{array}$}} & 19.4 & 8.43 & 21.0 & 17.5 & 22.8 & 15.0 & 26.0 & 18.0 \\
\hline & & 142 & 19.1 & 175 & 110 & 245 & 39.6 & 305 & 100 \\
\hline \multirow{2}{*}{$\begin{array}{l}\text { Vol.sêmen } / \text { peso } \\
(\mathrm{ml} / \mathrm{kg})\end{array}$} & não hip. & 0.67 & 57.5 & 1.20 & 0.31 & 1.12 & 65.3 & 2.00 & 0.34 \\
\hline & hipof. & 1.12 & 69.9 & 2.41 & 0.31 & 1.89 & 59.4 & 3.00 & 0.35 \\
\hline \multirow{2}{*}{$\begin{array}{l}\text { Turbilhonamento } \\
\text { (1 a } 5)\end{array}$} & não hip. & 1.8 & 120.4 & 5 & 0 & 3.25 & 72.7 & 5 & 0 \\
\hline & hipof. & 2.2 & 117.7 & 5 & 0 & 4.50 & 12.8 & 5 & 4 \\
\hline \multirow{2}{*}{$\begin{array}{l}\text { Sptz vivos } \\
(\%)\end{array}$} & não hip. & 94.4 & 3.1 & 98 & 91 & 96.3 & 2.1 & 99 & 94 \\
\hline & hipof. & 95.4 & 4.5 & 99 & 89 & 96.8 & 1.8 & 99 & 96 \\
\hline \multirow{2}{*}{$\begin{array}{l}\text { Concentração } \\
\left(10^{\circ} \mathrm{sptz} / \mathrm{ml}\right)\end{array}$} & não hip. & 11.8 & 21.7 & 15.3 & 8.6 & 27.8 & 3.0 & 28.7 & 27.1 \\
\hline & hipof. & 20.6 & 11.8 & 23.0 & 17.3 & 25.7 & 6.8 & 27.4 & 23.3 \\
\hline \multirow{2}{*}{$\begin{array}{l}N^{\circ} \text { sptz vivos/peso } \\
\left(10^{9} \mathrm{sptz} / \mathrm{kg}\right)\end{array}$} & não hip. & 8.63 & 74.5 & 15.4 & 2.6 & 30.4 & 64.3 & 54.4 & 8.4 \\
\hline & hipof. & 22.7 & 82.0 & 54.8 & 4.7 & 47.75 & 60.7 & 76.0 & 8.1 \\
\hline
\end{tabular}

x: médias; CV: coeficiente de variação; Máx.: máximo; Mín.: mínimo 
Tabela 2 - Médias dos parâmetros espermáticos nos curimbatás

\begin{tabular}{|c|c|c|c|c|c|}
\hline ' & & \multicolumn{2}{|c|}{ Ausência da fêmea } & \multicolumn{2}{|c|}{ Presença da fêmea } \\
\hline $\begin{array}{l}\text { Comprimento }(\mathrm{cm}) \\
\text { Peso }(\mathrm{g})\end{array}$ & & & & & \\
\hline $\begin{array}{l}\text { Vol.sêmen } / \text { peso } \\
(\mathrm{ml} / \mathrm{kg})\end{array}$ & $\begin{array}{l}\text { não hip. } \\
\text { hipof. }\end{array}$ & $\begin{array}{l}0.67 \\
1.12\end{array}$ & $\begin{array}{l}\text { ns } \\
\text { ns }\end{array}$ & $\begin{array}{l}1.12 \\
1.89\end{array}$ & $\begin{array}{l}\text { ns } \\
\text { ns }\end{array}$ \\
\hline $\begin{array}{l}\text { Turbilhonamento } \\
\text { (1 a 5) }\end{array}$ & $\begin{array}{l}\text { não hip. } \\
\text { hipof. }\end{array}$ & $\begin{array}{l}1.80 \\
2.20\end{array}$ & $\begin{array}{l}\text { ns } \\
\text { ns }\end{array}$ & $\begin{array}{l}3.25 \\
4.50\end{array}$ & $\begin{array}{l}\text { ns } \\
\text { ns }\end{array}$ \\
\hline $\begin{array}{l}\text { Sptz vivos } \\
(\%)\end{array}$ & $\begin{array}{l}\text { não hip. } \\
\text { hipof. }\end{array}$ & $\begin{array}{l}94.40 \\
95.40\end{array}$ & $\begin{array}{l}\text { ns } \\
\text { ns }\end{array}$ & $\begin{array}{l}96.30 \\
96.80\end{array}$ & $\begin{array}{l}\text { ns } \\
\text { ns }\end{array}$ \\
\hline $\begin{array}{l}\text { Concentração } \\
\left(10^{9} \mathrm{sptz} / \mathrm{ml}\right)\end{array}$ & $\begin{array}{l}\text { não hip. } \\
\text { hipof. }\end{array}$ & $\begin{array}{l}11.80 \\
20.60\end{array}$ & $\begin{array}{l}b \\
b\end{array}$ & $\begin{array}{l}27.80 \\
25.70\end{array}$ & $\begin{array}{l}\mathrm{a} \\
\mathrm{a}\end{array}$ \\
\hline $\begin{array}{l}N^{\circ} \text { sptz vivos/peso } \\
\left(10^{9} \mathrm{sptz} / \mathrm{kg}\right)\end{array}$ & $\begin{array}{l}\text { não hip. } \\
\text { hipof. }\end{array}$ & $\begin{array}{r}8.63 \\
22.70\end{array}$ & $\begin{array}{l}b \\
b\end{array}$ & $\begin{array}{l}30.40 \\
47.75\end{array}$ & $\begin{array}{l}\mathrm{a} \\
\mathrm{a}\end{array}$ \\
\hline
\end{tabular}

Letras diferentes representam diferenças significativas entre peixes na presença ou ausência da fêmea; ns: diferença não significativa ao nível de $5 \%$ de probabilidade pelo teste $\mathrm{F}$

\section{Abstract \\ Influence of female presence on seminal characteris- tics of curimbatá (Prochilodus marggravii Walbaum, 1972)}

This research was carried out to verify the influence of female presence on the seminal production on curimbatá fish. Nine males with high seminal production, and one female with high degree of gonadal maturity, were selected. Five males (average body weight of $142 \pm 27.1 \mathrm{~g}$ and average body length of $22.8 \pm 3.42 \mathrm{~cm}$ ) were placed in tank $A$ and four males (average body weight of $245 \pm 97 \mathrm{~g}$ and average body length of $22.8 \pm 3.42 \mathrm{~cm}$ ) and the female were placed in tank B. Two-hundred fifty hours-degree later, the semen was collected and analyzed. After that, the males received crude pituitary extract injection. After 250 hours-degree, a new seminal collection and analysis were performed. The female presence influenced $(p<0.05)$ sperm concentration and number of alive spermatozoa per $\mathrm{kg}$ of fish weight. Swirling, percent alive sperm and seminal volume per $\mathrm{kg}$ of fish weight were not significantly influenced by the presence of the female.

Key words: sperm; semen; fish; Prochilodus marggravii; female influence

\section{Agradecimentos}

Ao CNPq e à Universidade Federal de Viçosa pelo auxílio finaceiro.

\section{Referências bibliográficas}

Billard R, Bieniartz K, Popek W, Epler P, Saad A 1989. Observations on a possible pheromonal stimulation of milt production in carp (Cyprinus carpio L.). Aquaculture 77: 387392.

Blom E 1950. A one minute live-dead sperm stain by means of Eosin-Nigrosin. Fertility and Sterility 1(2): 176-177.

Carosfield J, Harvey B, Silveira WF, Kavamoto ET, Ramos SW, Silveira AN 1990. Criopreservação do sêmen de pacu, Piaractus mesopotamicus Holmeberg 1987. Bol Tec CEPTA 3: 1-4.

Coser AM, Godinho H, Ribeiro DM 1984. Cryogenic preservation of spermatozoa from Prochilodus scrofa e Salminus maxilosus. Aquaculture 17: 387-390.

Coser AM, Godinho H, Torquato VC 1987. Criopreservação de sêmen do peixe piau Leporinus silvestrii (Boulenger, 1902). Arq Bras Med Vet Zoot 39: 37-42.

Defraipont M, Sorensen PW 1991. Exposedure to the pheromone $17 \alpha, 20 \beta$-dihydroxy-4-pregnen-3-one enhances the behavioral spawning sucess, sperm production, and sperm motility of male goldfish. Anim Behav 46: 245-256.

Fribourg $\mathrm{JH}$ 1966. The aplication of differencial staining method to low-temperature studies on gold-fish spermatozoa. Progressive fish culturist 28 (4): 227-231.

Fonseca VO, Valle Filho FR, Abreu JJ, Mies Filho A 1992. Procedimentos para exame andrológico e avaliação de sêmen animal. Colégio Brasileiro de Reprodução Animal, Belo Horizonte, $69 \mathrm{pp}$.

Hancock JL 1957. The morphology of boar spermatozoa. J Roy Micro Soc 76: 84.

Kavamoto ET, Silveira WF, Godinho HM, Romagosa E 1989. Conservação em nitrogênio líquido do sêmen de Prochilodus scrofa Steindachner 1881. Bol Inst Pesca 16 (1): 29-36.

Pimentel Gomes F 1966. Curso de Estatística Experimental, $3^{a}$ ed., Escola Superior de Agricultura "Luiz de Queiroz", USP, Piracicaba, 404 pp.

Silveira WF, Kavamoto ET, Narahara MY 1985. Avaliação da qualidade e criopreservação em forma de "pellets" do sêmen de bagre, Rhamdia hilarii (Vallenciennes, 1840). Bol Inst Pesca 12: 7-11. 
Silveira WF, Kavamoto ET, Rigolino MG, Tabata YA 1987. O método espectrofotométrico na avaliação da concentração de espermatozóides na truta arco-íris, Salmo irideus Gibbons. B Inst Pesca 14: 69-73.

Silveira WF, Kavamoto ET, Rigolino MG, Tabata YA 1988. Fertilidade do sêmen da truta arco-íris, Salmo irideus Gibbons, em diferentes concentrações de espermatozóides por óvulo. B Inst Pesca 15 (1): 51-57.

Sorensen PW, Scott AP, Stacey NE, Bowdin L 1995. Sulfated 17,20 B-Dihydroxy-4pregnen-3-one functions as a potent and specific olfatory stimulant with pheromonal actions in the goldfish. Gen Comparative Endocrinology 100: 128-142.
Stacey N, Zheng W-b, Cardwell J 1994. Milt production in common carp (Cyprinus carpio): stimulation by a goldfish steroid pheromone. Aquaculture 127: 265-276.

Valencia O, Puentes R 1989. El Cultivo de la Cachama em Colombia. In RA Hernandez Cultivo de Colossoma. Bogotá, Colombia.

Weerd JHV, Richter CJJ 1991. Sex pheromones and ovarian developmente in Teleost fish (Mini Review). Comp Biochem Physiol 100A (3): 517-527.

Woynarovich E, Horvath L 1983. A propagação artificial de peixes de águas tropicais. FAO/CODEVASF/CNPq, Brasília, $200 \mathrm{pp}$ 